\title{
Phosphorylation of eIF2 $\alpha$ attenuates statin-induced apoptosis by inhibiting the stabilization and translocation of p53 to the mitochondria
}

\author{
SANG KYU LEE and YOUNG SANG KIM \\ Department of Biochemistry, College of Natural Sciences, \\ Chungnam National University, Daejeon 305-764, Republic of Korea \\ Received November 8, 2012; Accepted December 17, 2012
}

DOI: $10.3892 /$ ijo.2013.1792

\begin{abstract}
Statins are effective cholesterol-lowering drugs that exert pleiotropic effects, including cytotoxicity to cancer cells. We previously reported that simvastatin triggered the mitochondrial apoptotic pathway in MethA fibrosarcoma cells, which was accompanied by the translocation of stabilized p53 to the mitochondria. In this study, we investigated whether statins induce the endoplasmic reticulum (ER) stress response and the mechanisms by which this response is linked to the stabilization of p53 and its translocation to the mitochondria. Statins induced typical ER stress-related proteins, such as BiP/78 kDa glucose-regulated protein (Grp78) and CCAAT/ enhancer-binding protein homologous protein (CHOP), as well as the phosphorylation of protein kinase RNA-like endoplasmic reticulum kinase (PERK), eIF2 $\alpha$ and JNK. The statin-induced phosphorylation of eIF2 $\alpha$ and JNK was inhibited by supplementation with components of the mevalonate pathway, such as mevalonate, farnesyl pyrophosphate (FPP) and geranylgeranyl pyrophosphate (GGPP). Salubrinal, an inhibitor of the dephosphorylation of eIF $2 \alpha$, suppressed the loss of mitochondrial membrane potential and the translocation of stabilized p53 and Bax to the mitochondria; however, SP600125, a JNK kinase inhibitor, did not exert this effect. Furthermore, the eIF $2 \alpha$ knockdown sensitized cells to simvastatin-induced apoptosis and the overexpression of a non-phosphorylatable eIF $2 \alpha$ mutant [serine 51(Ser51)/alanine] enhanced the stabilization of p53 and its translocation to the mitochondria in response to simvastatin treatment. Taken together, these data indicate that eIF2 $\alpha$ phosphorylation in the context of the ER stress response plays a role in cell survival by counteracting the p53-mediated mitochondrial apoptosis in response to statins.
\end{abstract}

Correspondence to: Professor Young Sang Kim, Department of Biochemistry, College of Natural Sciences, Chungnam National University, 220 Gung-dong, Yuseong-gu, Daejeon 305-764, Republic of Korea

E-mail: young@cnu.c.kr

Key words: statins, endoplasmic reticulum stress, apoptosis, p53, eIF $2 \alpha$

\section{Introduction}

Statins inhibit $\beta$-hydroxy- $\beta$-methylglutaryl CoA (HMG-CoA) reductase, which converts $\mathrm{HMG}-\mathrm{CoA}$ to mevalonate. They are effective cholesterol-lowering drugs and exhibit anticancer effects by inducing apoptosis and cell cycle arrest (1). Moreover, the inhibition of the mevalonate pathway by statins causes perturbation of the endoplasmic reticulum (ER) and stress. In response to ER dysfunction, cells combat the stress and restore ER homeostasis by means of the unfolded protein response (UPR), which includes ER-associated degradation and control of translation (2,3). Among various ER responses, eIF $2 \alpha$ phosphorylation primarily protects cells from stress by attenuating global translation and specifically upregulating chaperone proteins, although under prolonged and severe stress it leads to apoptosis (4). Statin-induced eIF2 $\alpha$ phosphorylation has been shown to protect macrophages from hypoxia-induced cell death (5); however, lovastatin-induced eIF2 $\alpha$ phosphorylation has been shown to lead to apoptosis in human head and neck squamous cell carcinoma (6). Elucidating the role of eIF $2 \alpha$ phosphorylation induced by statins may lead to the development of novel protective and therapeutic approaches against hypercholesterolemia and cancer.

Under various stress conditions, the tumor suppressor p53 plays a pivotal role in the execution of ER stress-induced apoptosis via the activation of the $\mathrm{BH} 3$-only proteins, such as Puma and Noxa, in a transcription-dependent manner (7) and via a transcription-independent pathway; it activates members of the pro-apoptotic Bcl-2 family, such as Bax, Bid and Bak, or their translocation to the mitochondrial membrane (8). In our previous study, we demonstrated that simvastatin induced apoptosis in cancer cells by stabilizing p53 and stimulating its translocation with Bax to the mitochondria, resulting in the release of cytochrome $c$ (9). However, the mechanisms by which the ER stress response, particularly eIF $2 \alpha$ phosphorylation, is linked to the p53-mediated mitochondrial apoptotic pathway in statin-induced apoptosis, have not been investigated.

In the present study, we investigated the molecular link between eIF $2 \alpha$ phosphorylation in the ER stress response and the p53 transcription-independent mitochondrial apoptotic pathway in the statin-induced apoptosis of MethA fibrosarcoma cells. We report that the eIF $2 \alpha$ phosphorylation on 
serine 51 (Ser51) of the ER stress response attenuates cell death by inhibiting the stabilization of p53 and its translocation to the mitochondria in statin-induced apoptosis.

\section{Materials and methods}

Cells and reagents. Mouse MethA fibrosarcoma cells were maintained in RPMI-1640 (Invitrogen, Carlsbad, CA, USA) supplemented with $5 \%$ fetal bovine serum, $100 \mathrm{U} / \mathrm{ml}$ penicillin and $10 \mu \mathrm{g} / \mathrm{ml}$ streptomycin at $37^{\circ} \mathrm{C}$ and $5 \% \mathrm{CO}_{2}$. Simvastatin and lovastatin (MSD Korea, Ansan, Korea) were reconstituted in absolute ethanol and stored at $-20^{\circ} \mathrm{C}$. Mevalonolactone, farnesyl pyrophosphate (FPP) and geranylgeranyl pyrophosphate (GGPP) were purchased from Sigma-Aldrich (St. Louis, MO, USA). Salubrinal, tumnicamycin and SP600125 were obtained from Calbiochem (San Diego, CA, USA) and antitubulin antibody (T5186) from Sigma-Aldrich. Antibodies against p53, Bax, protein kinase RNA-like endoplasmic reticulum kinase (PERK), phospho-PERK, eIF2 $\alpha$, phosphoeIF2 $\alpha$, CCAAT/enhancer-binding protein homologous protein (CHOP)/GADD153, BiP/78 kDa glucose-regulated protein (Grp78), HRP-conjugated goat anti-mouse antibody and HRP-conjugated goat anti-rabbit antibody were supplied by Santa Cruz Biotechnology (Santa Cruz, CA, USA), while antibodies against phospho-JNK, total JNK and heat-shock protein (HSP) 60 were obtained from BD Biosciences (San Diego, CA, USA).

Cell fractionation and western blot analysis. Cell fractionation was performed with a Mitochondria Isolation kit (Pierce, Rockford, IL, USA) according to the manufacturer's instructions. For western blot analysis, cells were harvested, washed with ice-cold PBS and lysed in RIPA buffer [10 mM Tris ( $\mathrm{pH} 7.4$ ), $150 \mathrm{mM} \mathrm{NaCl}, 0.5 \%$ NP-40, 0.1\% deoxycholate, $1 \mathrm{mM}$ PMSF, $2 \mathrm{mM}$ sodium fluoride and $1 \mathrm{mM}$ sodium orthovanadate] for $15 \mathrm{~min}$. Samples (3-30 $\mu \mathrm{g}$ ) were then subjected to SDS-PAGE and western blot analysis was performed using primary antibodies and HRP-conjugated secondary antibodies, followed by detection with West-Pico Chemiluminescent Substrates (Pierce) in the dark.

Knockdown experiments and site-directed mutagenesis. siRNAs directed against eIF2 $\alpha$ and CHOP were purchased from Santa Cruz Biotechnology and transiently transfected into MethA cells using Lipofectamine 2000 (Invitrogen). Stable clones were selected in the presence of $4 \mu \mathrm{g} / \mathrm{ml}$ puromycin and screened by western blot analysis or RT-PCR. Substitution of the residue serine 51 of $\operatorname{IF} 2 \alpha$ with alanine (Ala) was performed using the QuikChange ${ }^{\text {TM }}$ Site-Directed Mutagenesis kit (Stratagene, La Jolla, CA, USA). Briefly, wild-type eIF $2 \alpha$ was amplified from MethA cDNA by PCR and cloned into the EcoRI and XhoI sites of pBluescript SK(+) vector (Stratagene). The mutant form of eIF2 $\alpha$ was amplified from wild-type eIF2 $\alpha$ /pBluescript SK(+) with Pfu polymerase (Intron, Seongnam, Korea) using the following mutagenesis primers and PCR conditions: forward, 5'-gcg aat tca tgc cgg ggc taa gtt gta g-3'; reverse, $5^{\prime}$-cgc tcg agt taa tct tca gct ttg gct t- 3 ; 18 cycles of $30 \mathrm{sec}$ at $95^{\circ} \mathrm{C}, 60 \mathrm{sec}$ at $55^{\circ} \mathrm{C}$ and $10 \mathrm{~min}$ at $68^{\circ} \mathrm{C}$. The PCR product was DpnI (Promega)-treated and transformed into DH5 $\alpha$ competent cells and the substitution was confirmed by DNA sequencing. The wild-type and mutant forms of eIF2 $\alpha$ were subcloned into the EcoRI and XhoI sites of the pCMV-tag2b vector (Stratagene) and transfected into the MethA cells. Stable clones of the eIF $2 \alpha$ wild-type and mutant forms were selected in the presence of $4 \mathrm{mg} / \mathrm{ml}$ neomycin and screened by western blot analysis.

Measurement of subgenomic content and mitochondrial membrane potential (MMP). To analyze subgenomic content, cells were incubated under the indicated conditions, washed with PBS, fixed with $70 \%$ ice-cold ethanol at $4^{\circ} \mathrm{C}$ for $1 \mathrm{~h}$ and stained with $50 \mu \mathrm{g} / \mathrm{ml}$ propidium iodide (PI) (Sigma-Aldrich) containing $100 \mu \mathrm{g} / \mathrm{ml}$ RNase (Sigma-Aldrich) at $37^{\circ} \mathrm{C}$ for 30 min. DNA content was analyzed by a FACSCalibur (Becton-Dickinson, San Jose, CA, USA). To analyze MMP, cells were washed with PBS and stained with $500 \mu 1$ of $4 \mu \mathrm{g} / \mathrm{ml}$ rhodamine- 123 solution at $37^{\circ} \mathrm{C}$ for $20 \mathrm{~min}$. They were then incubated with $0.1 \mathrm{mg} / \mathrm{ml}$ PI stock solution for $3 \mathrm{~min}$. Incorporation of rhodamine-123 and PI was analyzed by a FACSCalibur. The green fluorescence of rhodamine-123 and the red fluorescence of PI were collected over the range of FL1 and FL2, respectively. All data were calculated using CellQuest software (Becton-Dickinson).

Immunofluorescence. MethA cells were plated ( $1 \times 10^{5}$ cells) on poly-L-lysine-coated coverslips (BD Biosciences) and treated with the indicated reagents for 12 or 36 h. Following incubation, the cells were washed with PBS, stained with MitoTracker Red (Molecular Probes, Eugene, OR, USA) for $20 \mathrm{~min}$, washed twice with PBS, fixed, and permeabilized with Cytofix/Cytoperm solution (BD Biosciences) for $20 \mathrm{~min}$ at $4^{\circ} \mathrm{C}$. The cells were washed with PBS and blocked with PBS containing $1 \% \mathrm{BSA}$ for $20 \mathrm{~min}$. They were then incubated with primary antibodies (anti-p53 and anti-Bax) diluted in $1 \%$ BSA/PBS for $1 \mathrm{~h}$ at RT. After washing with PBS 5 times, cells were incubated with anti-rabbit conjugated Alexa 488 (Molecular Probes) for $1 \mathrm{~h}$ at RT and stained with $1 \mu \mathrm{g} / \mathrm{ml}$ Hoechst dye (Molecular Probes) for $10 \mathrm{~min}$. After washing with PBS, the coverslips were mounted using Vectashield HardSet (Vector Laboratories, Burlingame, CA, USA) on glass slides and analyzed under a laser scanning microscope (LSM 5; Carl Zeiss, Oberkochen, Germany).

\section{Results}

Statins induce apoptosis and ER stress response by depletion of the isoprenyl products of the mevalonate pathway. To evaluate the effect of statins on subgenomic content and MMP, we treated MethA fibrosarcoma cells with simvastatin (a natural statin) or lovastatin (a synthetic statin) for $24 \mathrm{~h}$ and analyzed DNA fragmentation and changes in MMP. As shown in Fig. 1, both simvastatin and lovastatin induced DNA fragmentation in a dose-dependent manner and disrupted MMP, while these effects were completely inhibited by supplementation with downstream products of the mevalonate pathway, such as mevalonate, FPP and GGPP. Supplementation with squalene, the direct precursor of cholesterol, was ineffective, indicating that the level of cholesterol was not critical for apoptosis (data not shown), but that the key factor was the depletion of isoprenoids of the mevalonate pathway. To investigate whether 
A

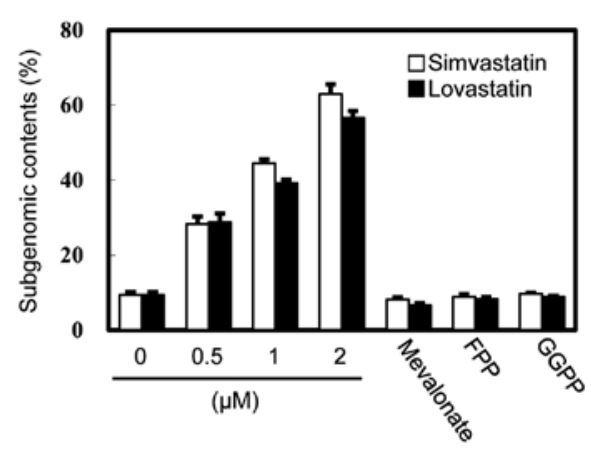

B

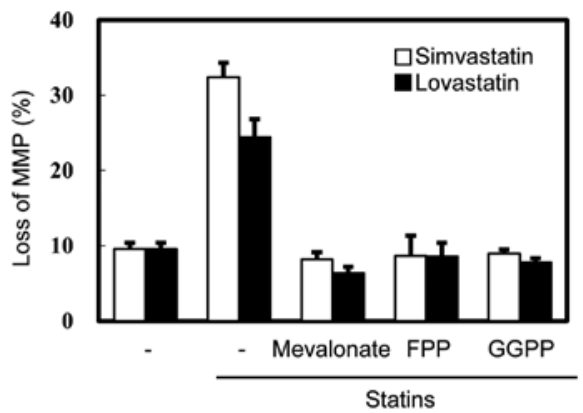

C

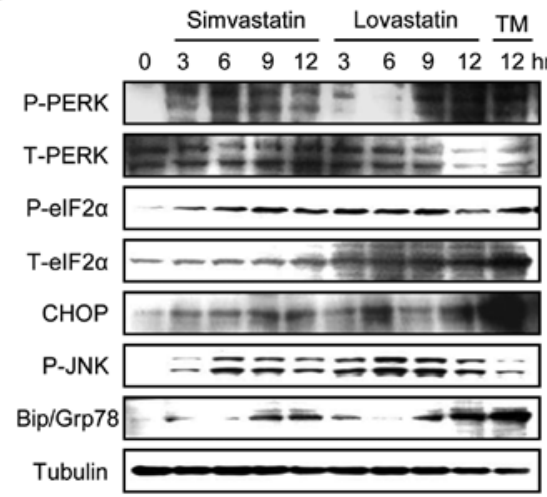

D

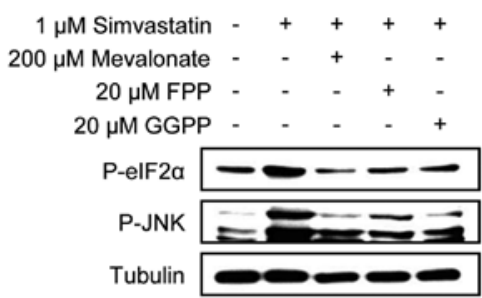

Figure 1. Statins induce apoptosis and ER stress responses by inhibiting the mevalonate pathway. (A) MethA cells were exposed to $0.5-2 \mu \mathrm{M}$ simvastatin or lovastatin for $24 \mathrm{~h}$ or treated with mevalonate $(200 \mu \mathrm{M})$, FPP $(20 \mu \mathrm{M})$ and GGPP $(20 \mu \mathrm{M})$ with $1 \mu \mathrm{M}$ simvastatin or lovastatin for $24 \mathrm{~h}$ and DNA content was analyzed by flow cytometry following PI staining. (B) MethA cells were incubated under the indicated conditions for $16 \mathrm{~h}$ and relative fluorescence intensity was analyzed by flow cytometry following staining with rhodamine-123 to evaluate the loss of MMP. (C) MethA cells were treated with $1 \mu \mathrm{M}$ lovastatin or simvastatin for the indicated times. Whole-cell extracts were analyzed for phosphorylation of PERK and eIF2 $\alpha$, expression of CHOP and Bip/Grp78 by western blot analysis using antibodies against phospho-PERK, total PERK, phospho-eIF2 $\alpha$, total eIF2 $\alpha$, CHOP, phospho-JNK, BiP/Grp78 and tubulin. TM; $2 \mathrm{nM}$ tunicamycin. (D) MethA cells were treated with the indicated reagents for $9 \mathrm{~h}$ and assessed by western blot analysis with antibodies specific for phosphoeIF2 $\alpha$, phospho-JNK and tubulin.

A

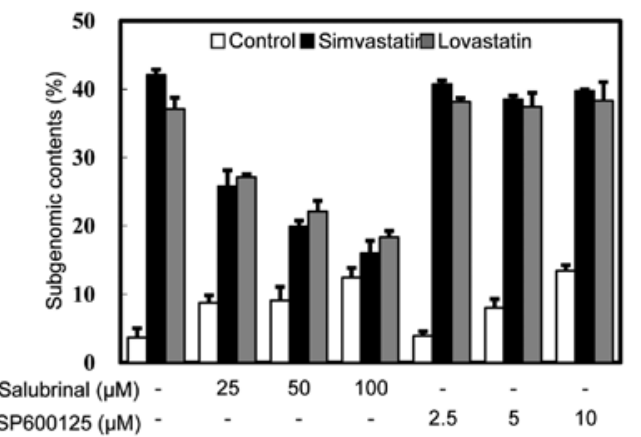

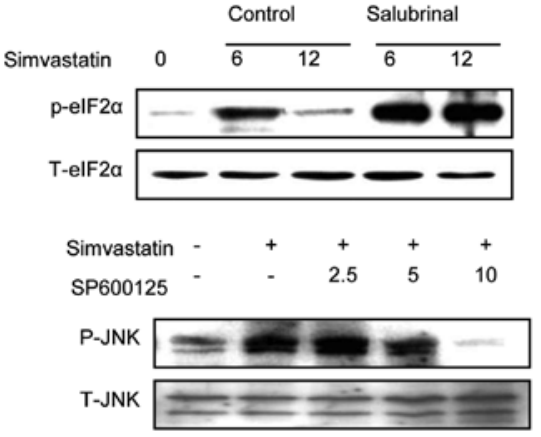

C

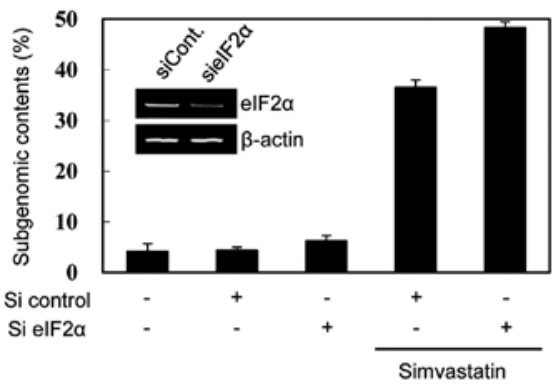

Figure 2. Inhibition of dephosphorylation of eIF2 $\alpha$ reduces statin-induced apoptosis. (A) MethA cells were pre-incubated with various concentrations of salubrinal (25-100 $\mu \mathrm{M})$, a selective inhibitor of eIF2 $\alpha$ dephosphorylation, or SP600125 (2.5-10 $\mu \mathrm{M})$, a specific inhibitor of JNK kinase, for 30 min and were then treated with $1 \mu \mathrm{M}$ simvastatin or lovastatin for $24 \mathrm{~h}$. Following incubation, the cells were stained with PI and subgenomic DNA content was assessed by flow cytometry. (B) MethA cells were pre-incubated with $50 \mu \mathrm{M}$ salubrinal for $30 \mathrm{~min}$ and then treated with $1 \mu \mathrm{M}$ simvastatin for the indicated times. Phosphorylation of eIF $2 \alpha$ was detected by western blot analysis using antibodies against phospho-eIF2 $\alpha$ (p-eIF2 $\alpha$ ) and total-eIF2 $\alpha$ (T-eIF2 $\alpha$ ). MethA cells were pre-incubated with 2.5-10 $\mu \mathrm{M} \mathrm{SP600125} \mathrm{for} 30 \mathrm{~min}$ and then treated with $1 \mu \mathrm{M}$ simvastatin for $6 \mathrm{~h}$. Phospho-JNK and total JNK were assessed by western blot analysis. (C) MethA cells were transfected with control (siCont.) or eIF2 $\alpha$-siRNA (sieIF2 $\alpha$ ) for $48 \mathrm{~h}$ and then treated with $1 \mu \mathrm{M}$ simvastatin for $24 \mathrm{~h}$. Following incubation, the cells were stained with PI and subgenomic DNA content was assessed by flow cytometry. Expression of eIF2 $\alpha$ mRNA was also measured by RT-PCR (inset). 
A

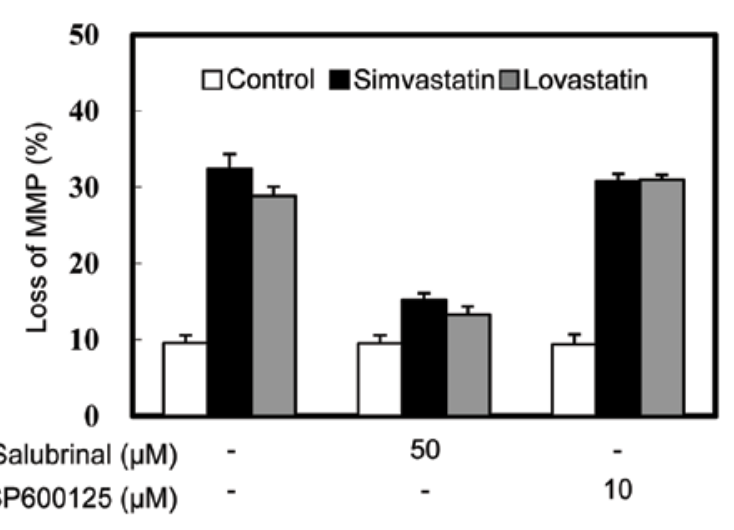

B

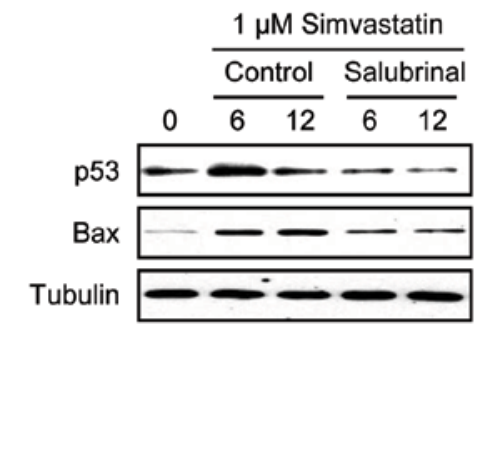

C

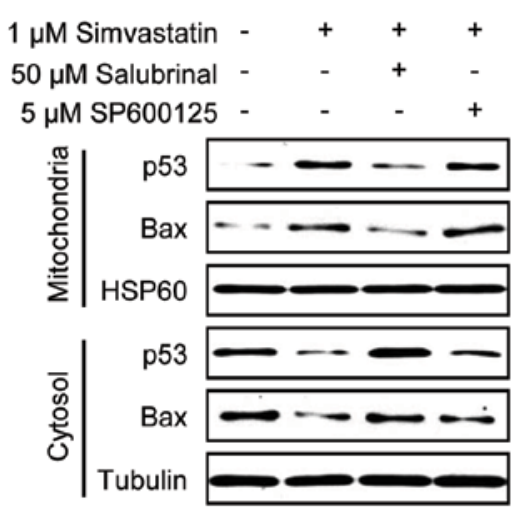

D

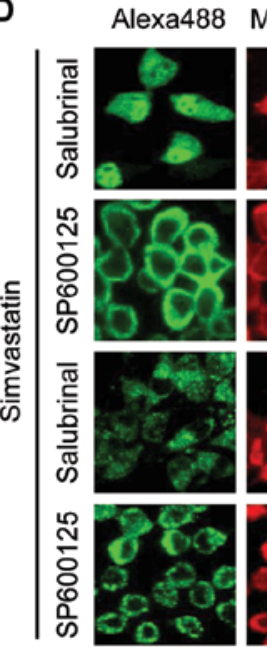

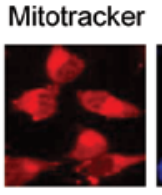
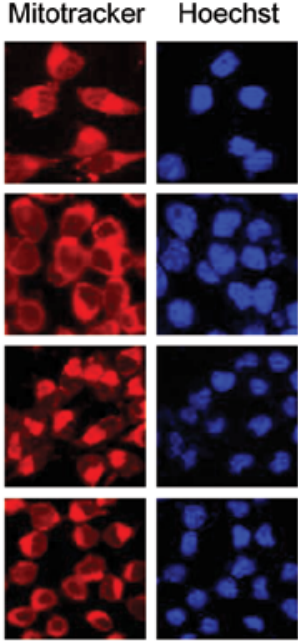

\section{Merge}
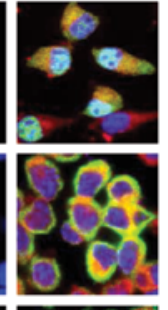

p53

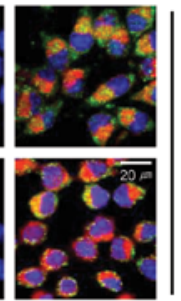

Figure 3. Inhibition of eIF2 $\alpha$ dephosphorylation by salubrinal decreases the stabilization and translocation of p53 to the mitochondria in simvastatin-induced apoptosis. (A) MethA cells pre-incubated with $50 \mu \mathrm{M}$ salubrinal or $10 \mu \mathrm{M}$ SP600125 for 30 min were treated with $1 \mu \mathrm{M}$ simvastatin or lovastatin for $16 \mathrm{~h}$. The cells were then stained with rhodamine-123 and relative fluorescence intensity was analyzed by flow cytometry to measure the loss of MMP. (B) MethA cells were pre-incubated with $50 \mu \mathrm{M}$ salubrinal for $30 \mathrm{~min}$ and were then treated with $1 \mu \mathrm{M}$ simvastatin for the indicated time periods. The level of p53 and Bax proteins were assessed by western blot analysis. (C) MethA cells were pre-incubated with $50 \mu \mathrm{M}$ salubrinal or $5 \mu \mathrm{M}$ SP600125 for 30 min and then treated with $1 \mu \mathrm{M}$ simvastatin for $12 \mathrm{~h}$. The cells were divided into cytosolic and mitochondrial fractions and the levels of p53 and Bax in the cytosolic and mitochondrial fractions were assessed by western blot analysis. (D) MethA cells were incubated under the indicated conditions for $12 \mathrm{~h}$ and immunostained with anti-p53 and anti-Bax antibody (green). p53 and Bax were observed under a confocal microscope at x630 magnification. Nuclear staining with Hoechst dye (blue) and mitochondrial staining with MitoTracker (red) are shown.

the statin-induced apoptosis was related to ER stress, we examined several cardinal indicators of ER stress in statin-treated MethA cells by immunoblot analysis (Fig. 1C). We discovered that the $\mathrm{CHOP}$ and $\mathrm{BiP} / \mathrm{Grp} 78$ protein expression was induced and that the phosphorylation of PERK, eIF2 $\alpha$ and JNK was increased. These results indicate that statins induce a general ER stress response during apoptosis.

To determine which downstream products of the mevalonate pathway are critical for statin-induced ER stress, we examined the effects of mevalonate, FPP and GGPP supplementation on simvastatin-treated MethA cells. As shown in Fig. 1D, these downstream products of the mevalonate pathway reduced the phosphorylation of eIF $2 \alpha$ and JNK, suggesting that the depletion of isoprenoids induced by statins disrupts ER homeostasis.

Inhibition of eIF $2 \alpha$ dephosphorylation opposes statin-induced apoptosis. To investigate whether the phosphorylation of eIF2 $\alpha$ and JNK induced by statins is involved in apoptosis, we assessed the effects of salubrinal (a selective inhibitor of eIF $2 \alpha$ dephosphorylation) and SP600125 (a specific inhibitor of JNK kinase). Notably, salubrinal treatment increased the phosphorylation of eIF2 $\alpha$ and reduced the DNA fragmentation induced by statins (Fig. 2A and B), whereas SP600125 treatment had no effect, although it clearly inhibited JNK phosphorylation (Fig. 2A and B). This result suggests that the phosphorylation of eIF $2 \alpha$, but not that of JNK, plays a role in statin-induced apoptosis. To confirm this finding, we investigated the effect of the eIF $2 \alpha$ knockdown on DNA fragmentation. As shown Fig. 2C, the MethA cells in which eIF2 $\alpha$ had been knocked down were more sensitive to simvastatin-induced apoptosis than the mocktransfected cells. Taken together, these results support the notion that the phosphorylation of eIF2 $\alpha$ negatively modulates statin-induced apoptosis in MethA cells.

Inhibition of eIF $2 \alpha$ dephosphorylation decreases the stabilization of p53 and its translocation to the mitochondria in statin-induced apoptosis. We previously reported that simvastatin activates the mitochondrial apoptotic pathway 
A

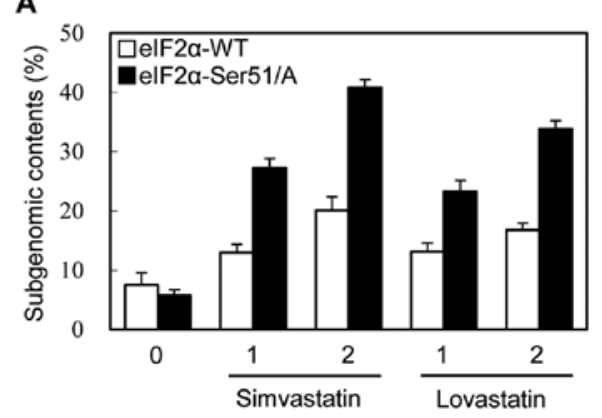

B

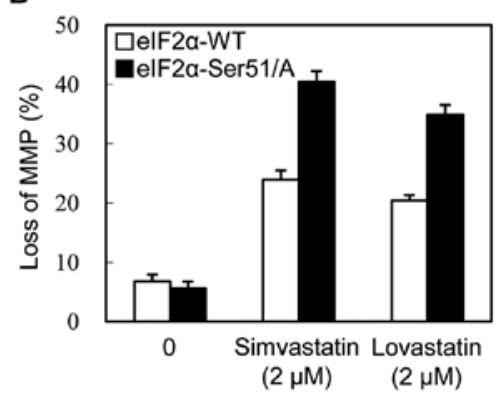

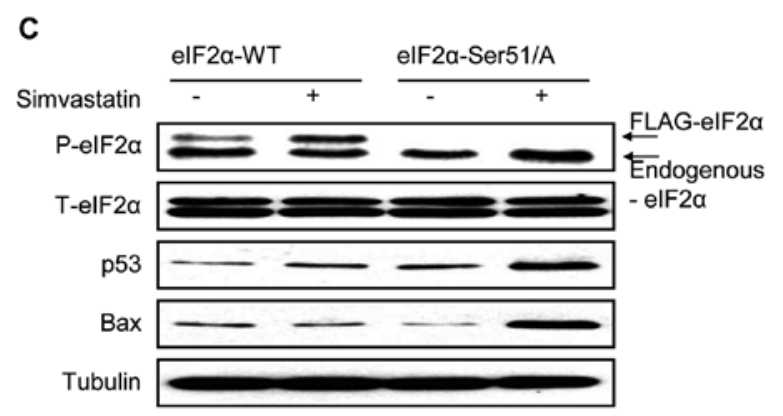

Figure 4. Phosphorylation of eIF2 $\alpha$ on serine 51 (Ser51) decreases the stabilization and translocation of p53 to the mitochondria in statin-induced apoptosis. MethA cells transfected with wild-type eIF2 $\alpha$ (eIF2 $\alpha$-WT) and cells transfected with the eIF2 $\alpha$ mutant (Ser51/A) were exposed to 1-2 $\mu$ M simvastatin or lovastatin for $24 \mathrm{~h}$. (A) Subgenomic DNA content and (B) loss of MMP were analyzed by flow cytometry. (C) MethA cells transfected with wild-type or mutant eIF $2 \alpha$ were treated with $1 \mu \mathrm{M}$ simvastatin for $9 \mathrm{~h}$. Phosphorylation of eIF2 $\alpha$ and expression of p53 and Bax were examined by western blot analysis.

in MethA cells accompanied by the stabilization of the p53 protein and its translocation with Bax to the mitochondria and that the knockdown of p53 expression decreases apoptosis (9). To determine whether the phosphorylation of eIF2 $\alpha$ and JNK contributes to the simvastatin-induced MMP loss and translocation of p53 to the mitochondria, we treated the cells with salubrinal (an inhibitor of the dephosphorylation of eIF $2 \alpha$ ) and SP600125 (a JNK kinase inhibitor). Salubrinal treatment significantly reduced statin-induced MMP loss (Fig. 3A) and the stabilization of $\mathrm{p} 53$ and Bax by simvastatin in the MethA cells (Fig. 3B), as well as the simvastatin-induced translocation of p53 and Bax to the mitochondria (Fig. 3C). By contrast, SP600125 had no discernible effect. The inhibitory effect of salubrinal on the simvastatin-induced translocation of p53 and Bax to the mitochondria was also evident via immunofluorescence staining (Fig. 3D). In the presence of salubrinal, the p53 and Bax mitochondrial co-localization was less apparent than in the presence of SP600125. These results demonstrate that the phosphorylation of eIF $2 \alpha$ counteracts the p53-mediated mitochondrial apoptotic pathway in simvastatin-induced apoptosis.

Phosphorylation of eIF $2 \alpha$ on Ser 51 is responsible for the stabilization of 53 and its translocation to the mitochondria. Several kinases, including PERK, phosphorylate eIF2 $\alpha$ on Ser51 in response to various ER stress inducers. To investigate whether the phosphorylation of eIF2 $\alpha$ on Ser51 is involved in the statininduced ER stress response and apoptosis, we examined the effect of the overexpression of the non-phosphorylatable mutant form of eIF2 $\alpha$ (Ser51/Ala) on simvastatin-induced apoptosis. The overexpression of the mutant eIF $2 \alpha$ (Ser51/Ala) attenuates the eIF $2 \alpha$ phosphorylation pathway (10). The flag-tagged wild-type eIF $2 \alpha$-transfected clone was used as the control. Both the DNA fragmentation and MMP loss induced by simvastatin were enhanced in the eIF $2 \alpha$ mutant-expressing clone compared to the wild-type-expressing clone (Fig. 4A and B). The effect of the overexpression of the eIF $2 \alpha$ mutant on p53 and Bax protein in response to simvastatin was also analyzed by immunoblot analysis. As shown in Fig. 4C, simvastatin treatment induced the phosphorylation of the flag-tagged eIF $2 \alpha$ wild-type protein, but not that of the flag-tagged mutant protein. At the same time, simvastatin effectively stabilized p53 and Bax proteins in the flag-tagged eIF $2 \alpha$ mutant clone compared with the flag-tagged eIF2 $\alpha$ wild-type clone (Fig. 4C). These results indicate that the phosphorylation of eIF2 $\alpha$ on Ser51 is responsible for the cell survival effect in the p53-mediated mitochondrial apoptosis of statin-treated MethA cells.

\section{Discussion}

Under stress conditions, p53 is stabilized and acts as a transcription factor for the expression of pro-apoptotic target genes, such as Puma, Noxa, Bax and Bid (11). In addition, cytoplasmic p53 directly activates the mitochondrial apoptotic pathway in a transcription-independent manner; i.e., p53 interacts with the Bcl-2 family members, Bcl-2 or Bcl-xL, leading to the translocation of Bax and Bid to the mitochondrial outer membrane (12). We previously reported that in response to statin, p53 itself is stabilized and translocated along with Bax to the mitochondria, thus activating the mitochondrial apoptotic pathway (9). In the present study, we demonstrate that statins induce the ER stress response in MethA cells by depleting the isoprenyl products of the mevalonate pathway and that the phosphorylation of eIF $2 \alpha$ on Ser51 plays a role in promoting cell survival. To our 
knowledge, this is the first study to demonstrate that the phosphorylation of eIF2 $\alpha$ counteracts the stabilization of p53 and its translocation to the mitochondria in statin-induced apoptosis.

Statins exert stress on cancer cells and display various cardinal features of ER stress response, which are pro- or anti-apoptotic. Lovastatin has been shown to induce apoptosis accompanied by reduced global protein translation by inducing eIF $2 \alpha$ phosphorylation. It has also been shown to induce general control non-repressed 2 (GCN2)-mediated activating transcription factor (ATF) 4 production followed by the increased expression of ATF3 and CHOP in a head and neck squamous cell carcinomas cell line (6). Lovastatin-induced apoptosis was attenuated in $\mathrm{CHOP}^{-/-}$and $\mathrm{GCN}^{-/-}$murine embryonic fibroblasts (MEFs), thus demonstrating the involvement of ATF4, CHOP and ATF3 in apoptosis. In multiple myeloma cells, lovastatin has been shown to induce $\mathrm{BiP} / \mathrm{Grp} 78$ and CHOP protein expression, the phosphorylation of eIF $2 \alpha$ and apoptosis, as well as the cleavage of poly(ADPribose) polymerase (PARP) (13). In contrast to these apoptotic effects of statin-induced ER stress, numerous studies have demonstrated that the ER stress response also attenuates the apoptotic response. Fluvastatin has been shown to induce BiP/Grp78 expression and to activate ATF6 and X-box binding protein-1 (XBP-1), but not CHOP and ATF4 in RAW264.7 cells (5). Among the induced proteins, the induction of $\mathrm{BiP} /$ Grp78 is responsible for the cytoprotective effect of fluvastatin pre-treatment against hypoxia-induced cell death. Simvastatin pre-treatment also exerted a neuroprotective effect by attenuating the ER stress response, with concomitant increases in ATF6 and XBP-1 protein expression during acute ischemia and reperfusion in rats (14). Recently, a novel protective effect of statins against atherosclerosis was proposed based on the finding that stearic acid-induced ER stress in macrophages was attenuated by statins (15). In sum, statins seem to exert differential ER stress responses depending on the strength, nature and duration of the stress. However, we observed that statins induce the majority of indicators of the ER stress response in MethA cells: the early phosphorylation of PERK, eIF $2 \alpha$ and JNK and the induction of CHOP and BiP/Grp78 protein expression.

The induction of CHOP and JNK phosphorylation suggests that, under our experimental conditions, ER stress in response to statins triggers apoptotic signals. The MethA clone in which CHOP was knocked down by siRNA exhibited increased resistance to statin-induced apoptosis, pointing to a pro-apoptotic role of CHOP (data not shown). However, the JNK inhibitor, SP600125 did not exert any effect on statin-induced apoptosis in our study (Figs. 2 and 3). The JNK kinase pathway is activated during lovastatin-induced apoptosis in the NB4 acute promyelocytic leukemia cell line (16) and human breast cancer cells (17). By contrast, the JNK pathway is downregulated (18) or not affected (19) in statin-induced apoptosis. Further study is required to elucidate the role of JNK phosphorylation in statin-induced ER stress.

Salubrinal, an inhibitor of eIF $2 \alpha$ dephosphorylation, clearly attenuated statin-induced apoptosis, suggesting that the phosphorylation of eIF $2 \alpha$ plays a role in triggering cell survival signals. Previous studies have also demonstrated that phosphorylation of eIF $2 \alpha$ protected cells under glucose deprivation stress $(20,21)$, and other ER stress (22). When global translation is inhibited by the phosphorylation of eIF2 $\alpha$, the translation of certain mRNAs, such as GCN2, ATF4 and $\mathrm{X}$-linked inhibitor of apoptosis protein (XIAP), is increased and this promotes tumor cell survival and chemoresistance $(23,24)$. In renal medullary cells exposed to urea stress, the phosphorylation of eIF $2 \alpha$ by activated GCN2 also exerts cytoprotective effects (25). On the other hand, there have been several reports demonstrating that the phosphorylation of eIF $2 \alpha$ is involved in apoptosis under various stress conditions, such as proteasome inhibition $(26,27)$, hypoxia (28) and tunicamycin (10). Moreover, salubrinal enhances cisplatin-induced nephrotoxicity (29). Depending on the nature and strength of the stress, the phosphorylation of eIF $2 \alpha$ seems to shift from its primary role of cytoprotection to apoptosis induction (4). More importantly, further studies are required to elucidate the signaling flows from the phosphorylation of eIF $2 \alpha$ to the reduced stability of p53 in response to statins. Unveiling the missing link between p53 stabilization and eIF $2 \alpha$ phosphorylation may contribute to the expansion of therapeutic approaches against hypercholesterolemia and cancer.

\section{Acknowledgements}

This study was supported by a grant from the Korea Healthcare Technology R\&D project, Ministry and Welfare, Republic of Korea (A084773).

\section{References}

1. Demierre MF, Higgins PD, Gruber SB, Hawk E and Lippman SM: Statins and cancer prevention. Nat Rev Cancer 5: 930-942, 2005

2. Harding HP, Zhang Y and Ron D: Protein translation and folding are coupled by an endoplasmic-reticulum-resident kinase. Nature 397: 271-274, 1999.

3. Hampton RY: ER-associated degradation in protein quality control and cellular regulation. Curr Opin Cell Biol 14: 476-482, 2002.

4. Holcik M and Sonenberg N: Translational control in stress and apoptosis. Nat Rev Mol Cell Biol 6: 318-327, 2005.

5. Chen JC, Wu ML, Huang KC and Lin WW: HMG-CoA reductase inhibitors activate the unfolded protein response and induce cytoprotective GRP78 expression. Cardiovasc Res 80: 138-150, 2008.

6. Niknejad N, Morley M and Dimitroulakos J: Activation of the integrated stress response regulates lovastatin-induced apoptosis. J Biol Chem 282: 29748-29756, 2007.

7. Li J, Lee B and Lee AS: Endoplasmic reticulum stress-induced apoptosis: multiple pathways and activation of p53-up-regulated modulator of apoptosis (PUMA) and NOXA by p53. J Biol Chem 281: 7260-7270, 2006.

8. Vaseva AV and Moll UM: The mitochondrial p53 pathway. Biochim Biophys Acta 1787: 414-420, 2009.

9. Lee SK, Kim YC, Song SB and Kim YS: Stabilization and translocation of p53 to mitochondria is linked to Bax translocation to mitochondria in simvastatin-induced apoptosis. Biochem Biophys Res Commun 391: 1592-1597, 2010.

10. Fritsch RM, Schneider G, Saur D, Scheibel M and Schmid RM: Translational repression of MCL-1 couples stress-induced eIF2 alpha phosphorylation to mitochondrial apoptosis initiation. J Biol Chem 282: 22551-22562, 2007.

11. Vousden $\mathrm{KH}$ and Lu X: Live or let die: the cell's response to p53. Nat Rev Cancer 2: 594-604, 2002.

12. Chipuk JE and Green DR: Dissecting p53-dependent apoptosis. Cell Death Differ 13: 994-1002, 2006.

13. Holstein SA and Hohl RJ: Isoprenoid biosynthetic pathway inhibition disrupts monoclonal protein secretion and induces the unfolded protein response pathway in multiple myeloma cells. Leuk Res 35: 551-559, 2011. 
14. Urban P, Pavliková M, Sivonová M, et al: Molecular analysis of endoplasmic reticulum stress response after global forebrain ischemia/reperfusion in rats: effect of neuroprotectant simvastatin. Cell Mol Neurobiol 29: 181-192, 2009.

15. Breder I, Coope A, Arruda AP, et al: Reduction of endoplasmic reticulum stress - a novel mechanism of action of statins in the protection against atherosclerosis. Atherosclerosis 212: 30-31, 2010.

16. Sassano A, Katsoulidis E, Antico G, et al: Suppressive effects of statins on acute promyelocytic leukemia cells. Cancer Res 67: 4524-4532, 2007.

17. Koyuturk M, Ersoz M and Altiok N: Simvastatin induces apoptosis in human breast cancer cells: p53 and estrogen receptor independent pathway requiring signalling through JNK. Cancer Lett 250: 220-228, 2007.

18. Campbell MJ, Esserman LJ, Zhou Y, et al: Breast cancer growth prevention by statins. Cancer Res 66: 8707-8714, 2006.

19. Wu J, Wong WW, Khosravi F, Minden MD and Penn LZ: Blocking the Raf/MEK/ERK pathway sensitizes acute myelogenous leukemia cells to lovastatin-induced apoptosis. Cancer Res 64: 6461-6468, 2004.

20. Scheuner D, Song B, McEwen E, et al: Translational control is required for the unfolded protein response and in vivo glucose homeostasis. Mol Cell 7: 1165-1176, 2001.

21. Muaddi H, Majumder M, Peidis P, et al: Phosphorylation of eIF2a at serine 51 is an important determinant of cell survival and adaptation to glucose deficiency. Mol Biol Cell 21: 3220-3231, 2010.
22. Boyce M, Bryant KF, Jousse C, et al: A selective inhibitor of eIF2alpha dephosphorylation protects cells from ER stress. Science 307: 935-939, 2005.

23. Thakor $\mathrm{N}$ and Holcik M: IRES-mediated translation of cellular messenger RNA operates in eIF2 $\alpha$-independent manner during stress. Nucleic Acids Res 40: 541-552, 2011.

24. Ron D and Walter P: Signal integration in the endoplasmic reticulum unfolded protein response. Nat Rev Mol Cell Biol 8: 519-529, 2007.

25. Cai $Q$ and Brooks HL: Phosphorylation of eIF2 $\alpha$ via the general control kinase, GCN2, modulates the ability of renal medullary cells to survive high urea stress. Am J Physiol Renal Physiol 301: F1202-F1207, 2011.

26. Jiang HY and Wek RC: Phosphorylation of the alpha-subunit of the eukaryotic initiation factor-2 (eIF2alpha) reduces protein synthesis and enhances apoptosis in response to proteasome inhibition. J Biol Chem 280: 14189-14202, 2005.

27. Schewe DM and Aguirre-Ghiso JA: Inhibition of eIF2alpha dephosphorylation maximizes bortezomib efficiency and eliminates quiescent multiple myeloma cells surviving proteasome inhibitor therapy. Cancer Res 69: 1545-1552, 2009.

28. Liu Y, László C, Liu W, Chen X, Evans SC and Wu S: Regulation of $\mathrm{G}(1)$ arrest and apoptosis in hypoxia by PERK and GCN2-mediated eIF2alpha phosphorylation. Neoplasia 12: 61-68, 2010.

29. Sayan BS, Sayan AE, Knight RA, Melino G and Cohen GM: p53 is cleaved by caspases generating fragments localizing to mitochondria. J Biol Chem 281: 13566-13573, 2006. 